\author{
Marquette University \\ e-Publications@Marquette
}

\title{
Theoretical investigation of quantum-dot avalanche photodiodes for mid-infrared applications
}

\author{
Sanjay Krishna \\ University of New Mexico - Albuquerque \\ Oh-Hyun Kwon \\ Air Force Research Laboratory \\ Majeed M. Hayat \\ Marquette University, majeed.hayat@marquette.edu
}

Follow this and additional works at: https://epublications.marquette.edu/electric_fac

Part of the Computer Engineering Commons, and the Electrical and Computer Engineering Commons

\section{Recommended Citation}

Krishna, Sanjay; Kwon, Oh-Hyun; and Hayat, Majeed M., "Theoretical investigation of quantum-dot avalanche photodiodes for mid-infrared applications" (2005). Electrical and Computer Engineering Faculty Research and Publications. 540.

https://epublications.marquette.edu/electric_fac/540 
Marquette University

e-Publications@Marquette

\section{Electrical and Computer Engineering Faculty Research and Publications/College of Engineering}

This paper is NOT THE PUBLISHED VERSION; but the author's final, peer-reviewed manuscript. The published version may be accessed by following the link in the citation below.

IEEE Journal of Quantum Electronics, Vol. 41, No. 12 (2005): 1468-1473. DOI. This article is (C) Institute of Electrical and Electronic Engineers (IEEE) and permission has been granted for this version to appear in e-Publications@Marquette. Institute of Electrical and Electronic Engineers (IEEE) does not grant permission for this article to be further copied/distributed or hosted elsewhere without the express permission from Institute of Electrical and Electronic Engineers (IEEE).

\section{Theoretical investigation of quantum-dot avalanche photodiodes for mid-infrared applications}

\section{Sanjay Krishna}

Center for High Technology Materials and the Department of Electrical and Computer Engineering, University of New Mexico, Albuquerque, NM

Oh-Hyun Kwon

Air Force Research Laboratory, Wright Paterson Air Force Base, $\mathrm{OH}$

Majeed M. Hayat

Department of Electrical and Computer Engineering, University of New Mexico, Albuquerque, NM

\section{Abstract}

A novel midinfrared sensor, called the quantum-dot avalanche photodiode (QDAP), is proposed which is expected to have improved signal-to-noise ratio (SNR) in the presence of Johnson noise over its quantum-dot (QD) counterpart. In the QDAP, an intersubband QD detector is coupled with a thin, low-noise GaAs avalanche layer through a tunnel barrier. The avalanche layer provides the necessary photocurrent gain required to 
overcome Johnson noise and nearly achieve the dark-current-limited SNR of the QD detector. In the proposed three-terminal device, the applied biases of the QD-detector and the avalanche-photodiode sections of the QDAP are controlled separately. This feature permits the control of the QDs responsivity and dark current independently of the operating avalanche gain, thereby allowing the optimization of the avalanche multiplication factor to maximize the photocurrent's SNR. Notably, a heterojunction potential-barrier layer can also be utilized to further improve the SNR. For example, when the standard deviation of the Johnson noise is four times greater than the dark current, calculations show that the SNR enhancement offered by an avalanche multiplication factor of 5 results in relaxing the cooling requirement from 20 to $80 \mathrm{~K}$.

\section{SECTION I. Introduction}

Mid-Infrared detectors are useful for a variety of applications ranging from laser aided ranging (LADAR), remote sensing of toxic chemical agents to chemical spectroscopy and vegetation and geological monitoring [1]. The important wavelength regime for operation of terrestrial sensors is in the two transmission windows of the atmosphere in midwave infrared (MWIR, 3-8 $\mu \mathrm{m}$ ) regime and the long-wave infrared (LWIR, 8-14 $\mu \mathrm{m}$ ) regime. Space-based sensors, such as those mounted on satellites and used for thermal imaging, usually operate in the very long wave infrared (VLWIR, $>14 \mu \mathrm{m})$ regime. However, one of the biggest problems plaguing quantum-dot (QD) detectors is their low quantum efficiency, which leads to a lower detectivity, responsivity and limits their operating temperature to about 70-80 K. If the operating temperature of QD detectors can be increased to 150$200 \mathrm{~K}$, fairly inexpensive Peltier coolers can be used. Presently, there are no photonic detectors that can operate in this temperature range [1]. A 100-K increase in the operating temperature would lead to a dramatic decrease in the cost and complexity of infrared imaging systems and would represent a major technological breakthrough. Moreover, as the operating wavelength is increased, the performance of a sensor deteriorates and, hence, the operating temperature must be decreased [1]. It is to be noted that the only photonic detectors that operate in the VLWIR regime do require cooling to $4.2 \mathrm{~K}$. This cooling requirement places an enormous constraint on an infrared sensors and imaging systems, thereby leading to an increase in complexity and cost.

In this paper, we propose a novel midinfrared sensor, named the quantum-dot avalanche photodiode (QDAP), in which an intersubband QD detector is coupled with an avalanche layer through a tunnel barrier. The tunnel barrier reduces the dark current while the avalanche section supplies the photocurrent with internal gain. This gain tends to combat Johnson noise, which would otherwise plague the signal-to-noise ratio (SNR) of ultraweak signals. Additionally, prior studies in our group have revealed that while the use of a heterojunction potentialbarrier layer simultaneously lowers the dark current and the photocurrent of the QD detector; the barrier has a larger impact on the dark current [2]. Thus, the dark-current-limited SNR, which is measured by the specific detectivity $(D *)$, is larger in the presence of the heterojunction potential-barrier layer. However, due to the reduced photocurrent, the SNR does not achieve its dark-current limit in the presence of Johnson noise. In the QDAP, the avalanche layer provides the necessary photocurrent gain required to amplify the photocurrent and elevate its SNR to the dark-current limit with a slight penalty, due to the excess noise brought about by avalanche multiplication. 


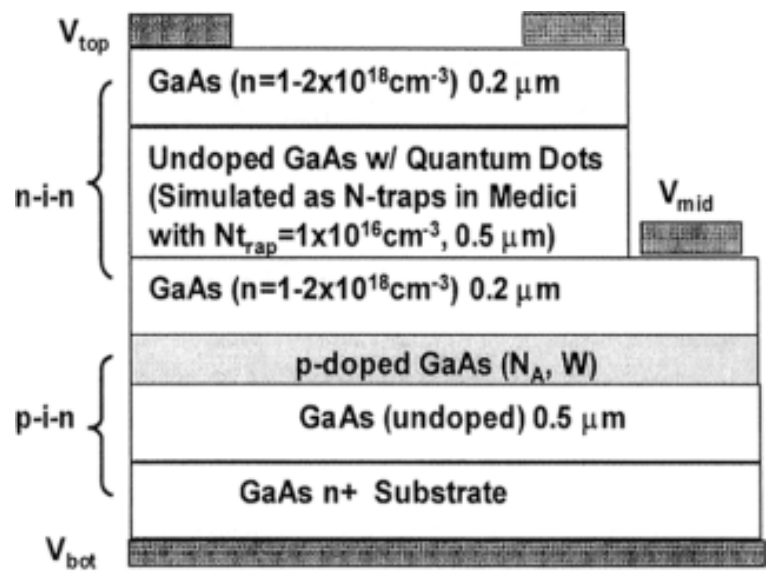

(a)

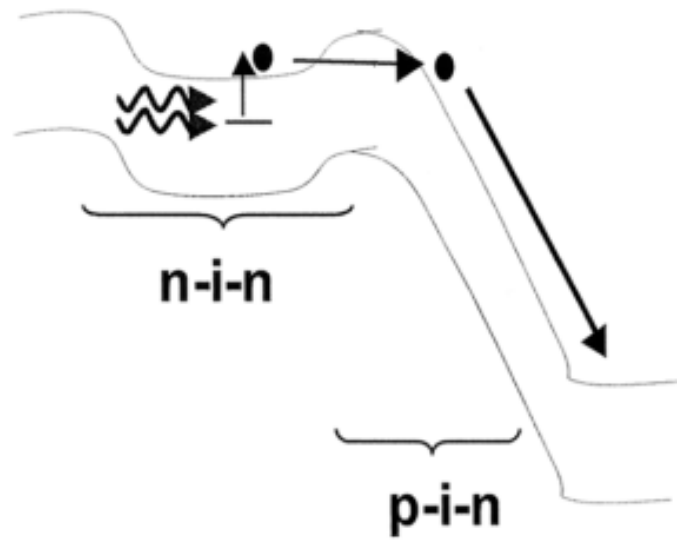

(b)

Fig. 1. (a) Schematic of the proposed QDAP. (b) Energy-band sketch illustrating its principle of operation. The photogenerated carriers in the $\mathrm{n}$-i-n section tunnel through the thin $\mathrm{p}$-doped layer and are multiplied in the high-field i-layer of the avalanche photodiode section.

In the proposed three-terminal device, the applied biases of the QD and the avalanche layer are controlled separately. This feature permits the control of the QDs responsivity and dark current independently of the operating avalanche gain, thereby allowing the optimization of the multiplication factor to maximize the photocurrent's SNR. Moreover, if a heterojunction potential-barrier layer is employed just before the avalanchephotodiode section, the thickness of the avalanche layer may also be optimized to minimize the excess noise factor. [3]

The higher SNR offered by the QDAP could be either used to obtain a higher sensitivity at the same temperature or to achieve a comparable performance at higher operating temperatures. The mode of operation would be dictated by the specific application. Additionally, the QDAP can be operated in a Geiger mode and used as a single-photon counter. In this direction, the fast response time of the QD detector could be utilized for gatedmode operation with a short on-cycle. This would reduce the number of dark carriers generated during the oncycle, which would potentially reduce the false-count rate thus enabling the detection of ultra weak signals. Presently there are no single photon sensors that operate beyond $2 \mu \mathrm{m}$. Additionally, by using a novel quantumdots-in-a-well (DWELL) design, we have recently reported a three-color detector operating in the MWIR, LWIR, and VLWIR regimes [4]. These designs, too, could be incorporated into the QDAP detector to realize multicolor detectors with high sensitivity. 


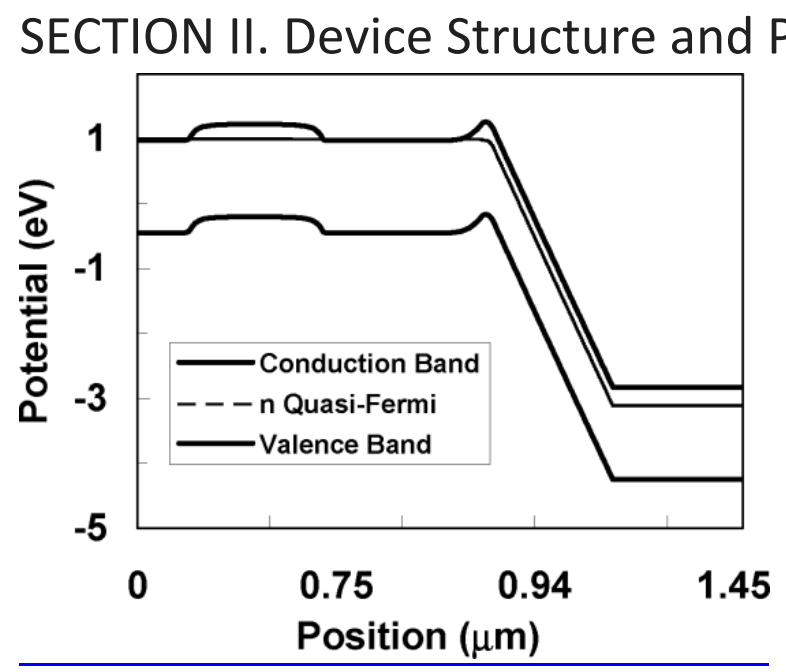

Fig. 2. Calculated bandstructure and electronic quasi-Fermi levels of the QDAP for $V$ top $=-1 \mathrm{~V}, V \mathrm{mid}=0 \mathrm{~V}$, and $V$ bot $=5 \mathrm{~V}$ for $p=5 \times 1018 \mathrm{~cm}-3$. The quantum dots are simulated as $\mathrm{N}$-level traps $300 \mathrm{meV}$ below the conductionband edge in accordance with the activation energy of the quantum dots.

In the proposed QDAP device, an n-i-n intersubband DWELL detector is grown on top of a $\mathrm{p}-\mathrm{i}-\mathrm{n}$ avalanche photodiode (APD) structure in a single-step epitaxy. The schematic of the proposed device (with the three contact regions) is shown in Fig. 1(a). During the device operation, the $n-i-n$ and the $p$-i-n regions of the device are both reverse biased (i.e., $V$ top $<V$ mid $<V$ bot). The photogenerated electrons are created in the $n$-i-n structure and are swept by the applied field toward the $\mathrm{p}$-i-n region. The thickness of the p-region is made fairly thin to allow the photoexcited electrons to tunnel into the intrinsic region of the reverse biased diode. Since the $p-i-n$ structure is biased in the punch-through, subbreakdown regime, there is a finite gain due to the avalanche multiplication process, which leads to an increase in the photocurrent. It is to be noted that the excess noise produced in the APD would be significantly lower than that produced in a conventional APD since there is a selective injection of electrons only and there are no primary holes involved in the avalanche process. (This is similar to the low-noise separate-absorption-multiplication (SAM) heterostructure APDs, which are specifically designed to achieve this property.) [5]

A sketch of the bandstructure of the QDAP is shown in Fig. 1(b). The crucial design parameters are: 1) the doping and thickness of the p-layer and 2) the intrinsic-layer thickness in the p-i-n layer. The latter controls the extent of the avalanche gain, excess noise factor, and breakdown characteristics of the APD. Modifications of the structure, such as inclusion of heterojunction potential-barrier layer (to be discussed in a later section), are expected to further lead to improved device performance. 


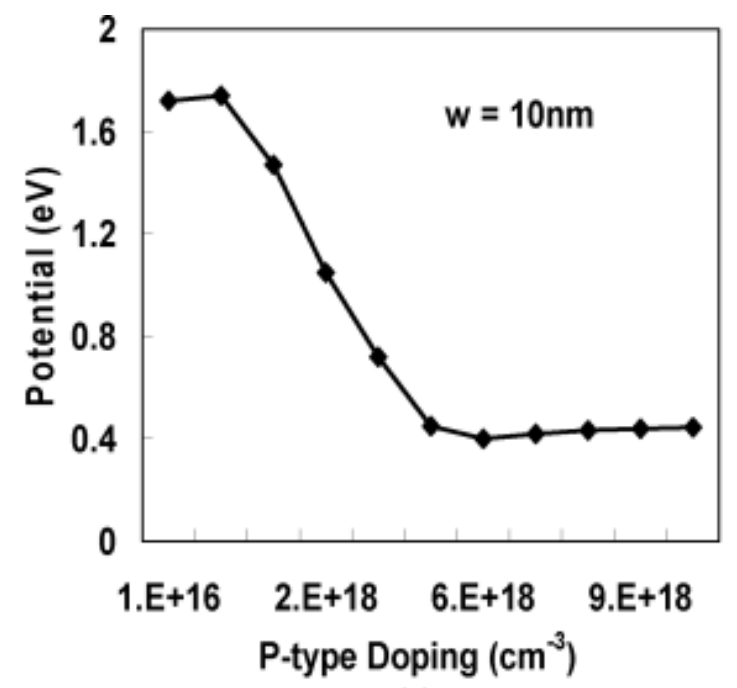

(a)

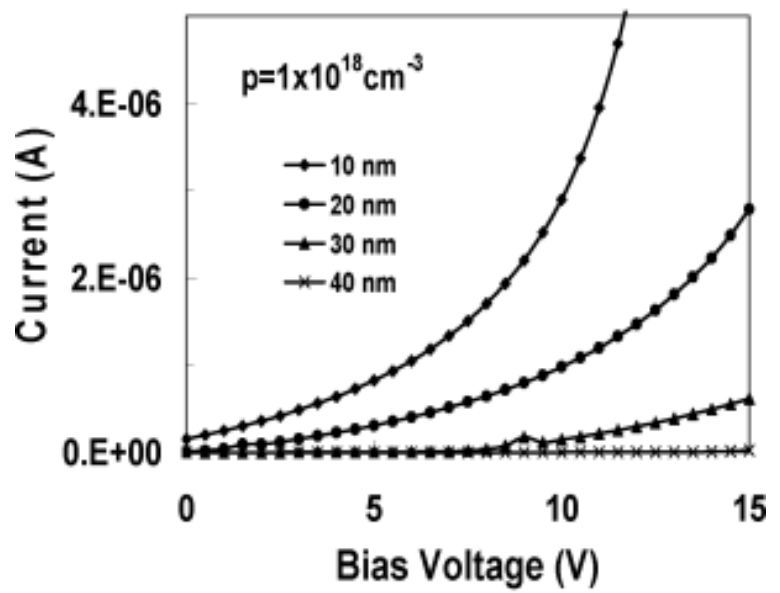

(b)

Fig. 3. (a) Variation of the barrier height as a function of the doping in the tunnel barrier. (b) Effect thickness of the barrier layer on the current-voltage characteristic of the QDAP for $p=1.0 \times 1018 \mathrm{~cm}-3$.

A careful analysis of the QDAP was undertaken using a commercially available simulation software (MEDICI). The quantum dots were simulated by N-traps with activation energy of $300 \mathrm{meV}$, in accordance with the position of ground state of the QD. In particular, the bandstructure and transport properties of the device were investigated for different doping levels and thickness of the p-type layer. Fig. 2 shows the calculated bandstructure of the QDAP along with the electronic quasi-Fermi levels for Vtop=-1 V, Vmid=0 V, and Vbot=5 Vfor $p=5 \times 1018 \mathrm{~cm}-3$. Note that the calculated bandstructure is in very good agreement with the proposed schematic shown in Fig. 1(b). Fig. 3(a) shows the barrier height, measured from the conduction band edge of GaAs, as a function of the doping of the p-type region. As the doping in the $p$-type region is increased the peak of the barrier moves from the intrinsic region of the $p$-i-n section to the $p$-type region, since the voltage drop across a highly doped layer becomes very small. For a p-type doping in the range $5 \times 1018-10 \times 1018 \mathrm{~cm}-3$, the potential barrier is approximately $400 \mathrm{meV}$.

The transport properties of the structure were studied by simulating the $I-V$ characteristics of the device. Fig. 3(b) shows the current collected at the bottom contact (which is indicative of the tunneling probability through the p-type barrier) as a function of the voltage, parameterized by the thickness of the p-type layer, doped at $1018 \mathrm{~cm}-3$. As expected, the tunneling probability increases with a decrease in the thickness of the barrier layer. The simulated $I-V$ characteristics of the QDAP suggest that, depending on the thickness and barrier-height 
of the p-type layer as well as the applied electric field, it is possible to have a significant fraction of carriers reach the high field multiplication region. A more important question is what fraction of photocurrent carriers (before thermalization) tunnel through and reach the high-field multiplication region. We are currently fabricating QDAP devices to experimentally determine this number. For the proposed structure, we believe that the optimal thickness of the p-type layer should be between $30-40 \mathrm{~nm}$ with a p-type doping of $\sim 0.5 \times 1019-1019 \mathrm{~cm}-3$. From Fig. 3, we observe that there is a good level of tolerance to the specified doping and thickness value, making the design fairly robust to fluctuations during growth.

Finally, one factor that may limit the carrier transport of the QDAP is the trapping of holes (resulting from the ionization process in the multiplication region) in the potential minimum near the junction. However, our understanding is that the holes that are trapped in the potential minimum may recombine with some of the electrons that are injected from the QDIP portion of the device.

\section{A. SNR Improvement due to Use of Heterojunction Potential-Barrier Layer}

The performance of the device can be further improved by replacing the p-type GaAs barrier with a p-type AlGaAs [2]. This has two advantages. Firstly, the height of the barrier can be controlled by varying the composition of the AIGaAs layer while keeping the doping fixed. Secondly, the AlGaAs barrier can serve to improve both the excess-noise and breakdown characteristics of the APD due to the so-called initial-energy effect [6]. In particular, the presence of a sharp electric-field gradient in the AlGaAs layer, near the GaAs interface, serves to energize the transported electrons prior to their injection in the GaAs intrinsic layer. Due to the high ionization-energy threshold of AIGaAs, the electrons do not ionize in the AlGaAs layer; however, they tunnel into the GaAs layer with a substantial energy. Consequently, these electrons may ionize in the GaAs layer after traveling a reduced dead space in GaAs as result of the energy already acquired by the electron while traveling the high-field gradient in the AIGaAs layer. Indeed, it has been argued recently that when the primary electrons that initiate the avalanche multiplication in the GaAs layer enter the layer "hot," the excess noise factor is reduced and the breakdown-probability characteristics are enhanced [3], [7]. For example, for $0.6 \mathrm{Al}$ composition, a reduction of $36 \%$ in the excess noise factor was observed as a result of the initial-energy effect [6] (an excess noise factor below 3 for a gain of 10).

In what follows we quantify the SNR improvement in QD detectors reported by Rotella et al. [2] as a result of the insertion of an AlGaAs potential-barrier layer. First, suppose that the insertion of the barrier layer results in a reduction in the dark current by a factor $1 / r$ where $r=i d o / i d$, id is the dark current for a device with a heterojunction-barrier layer, and ido is the dark current for a device without the heterojunctionbarrier layer (i.e., with a GaAs homojunction barrier) under identical operating conditions. Similarly, the reduction in the photocurrent reduction $\rho$ as a result of the insertion of the heterojunction barrier layer is defined as $\rho=i p / i p o$, where ip is the photocurrent with the heterojunction potential-barrier layer and ipo is the photocurrent without this layer. Clearly, the quantities $r$ and $\rho$ are bias-voltage dependent. The dark-current-limited SNR without the heterojunction-barrier layer is $\mathrm{SNR}_{0}=i_{p o}^{2} / 2 q\left(i_{p o}+i_{d o}\right) \Delta f$, where $\Delta f$ is the electrical bandwidth and $q$ is the electron charge, while the SNR with the heterojunction potential-barrier layer is $\mathrm{SNR}=i_{p}^{2} / 2 q\left(i_{p o}+i_{d}\right) \Delta f$. If we now assume that $i_{p o} \ll i_{d o}$ and $i_{p} \ll i_{d}$, as in the case of ultra-weak signal detection, we conclude that

$$
\frac{\mathrm{SNR}}{\mathrm{SNR}_{0}}=\rho^{2} r
$$




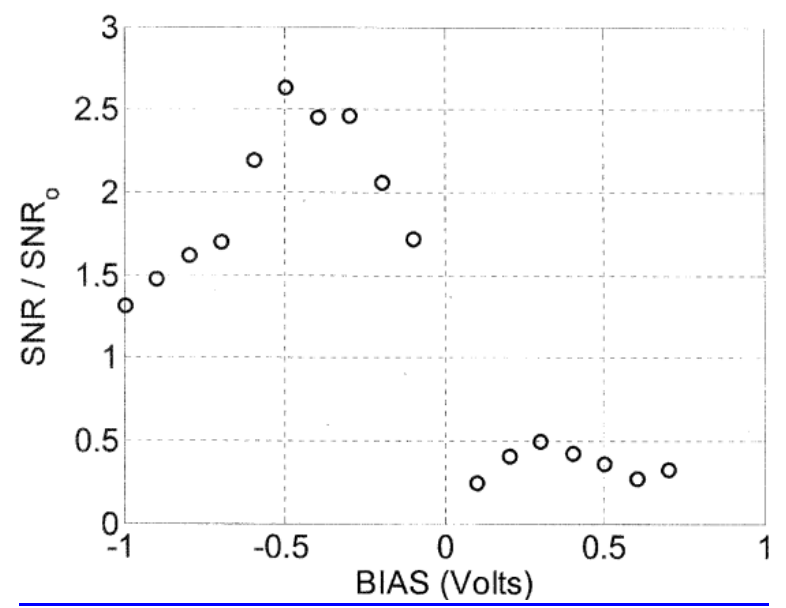

Fig. 4. SNR improvement offered by the insertion of a heterojunction potential-barrier layer as a function of applied bias. Data is obtained from Rotella et al. [2].

Rotella et al. [2] fabricated InAs-InGaAs DWELL detectors with and without the AIGaAs heterojunction potentialbarrier (or current-blocking) layer. The photocurrent reduction $\rho$ is obtained from the ratio of responsivity curves (with and without the heterojunction potential-barrier layer) as a function of applied bias at $78 \mathrm{~K}$. The dark current reduction, $1 / r$, is obtained from the ratio of dark current curves, both with and without the potential-barrier layer, reported in Rotella et al., [2] as a function of applied bias at $80 \mathrm{~K}$. (We have neglected the effect of the $2 \mathrm{~K}$ temperature difference.) Fig. 4 shows the measured SNR improvement, SNR/SNRO, as a function of applied bias. This shows an SNR enhancement in all cases of reverse biasing. For example, when the bias is $V$ top $-V$ mid $=-1 \mathrm{~V}$, the SNR enhancement is approximately $30 \%$. It is also seen that none of the forward biases render an SNR improvement. However, these forward biases (of the QDIP section) are irrelevant to the QDAP since reverse biasing the QDIP section is required to induce electron injection into the avalanche layer.

\section{SECTION III. Signal-to-Noise Ratio Improvement due Avalanche Gain}

In this section we calculate the SNR of the QDAP in the presence of Johnson noise and show the improvement offered by the avalanche gain, which limits the degrading effect of Johnson noise. In addition, as described in Section II.A, the dark-current-limited SNR can be improved in a QD detector with the insertion of a potentialbarrier layer. However, when the Johnson noise is not negligible, the presence of the barrier, despite its beneficial effect in the dark-current-noise limit, may result in a lower SNR, simply due to the combined effect of Johnson noise and the reduction in the responsivity. In order to maintain the SNR improvement offered by the heterojunction potential-barrier layer, the role of the Johnson noise must be suppressed through the avalanche gain. 


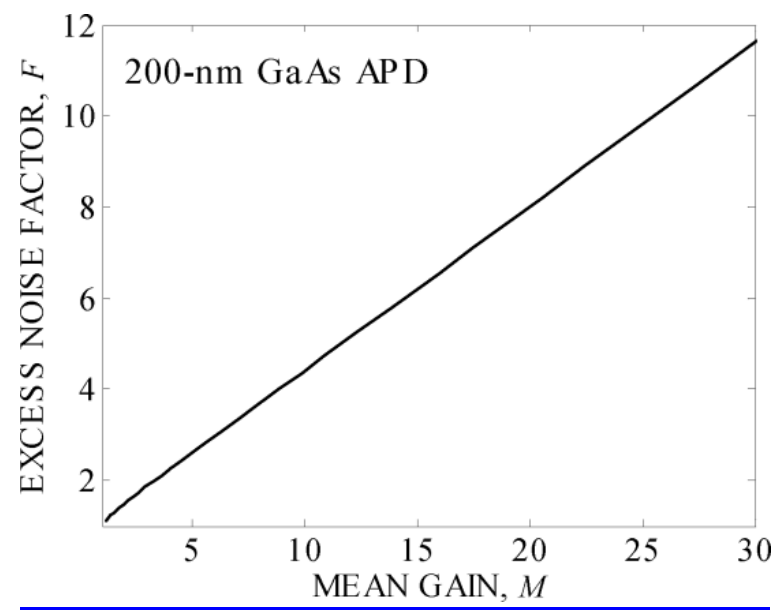

Fig. 5. Calculated excess noise factor $F$ as a function of the avalanche mean gain $M$ for a 200-nm GaAs avalanche multiplication layer. The curve was generated according to the dead-space multiplication theory [8].

The expression for the QDAP's SNR is similar to that for a photodiode and it is given by

$$
\mathrm{SNR}=\frac{I_{p}^{2}}{\sigma_{S}^{2}+\sigma_{T}^{2}}
$$

where

$$
\begin{aligned}
I_{p}^{2} & =\left(\mathrm{MRP}_{\mathrm{in}}\right)^{2} \\
\sigma_{S}^{2} & =2 q M^{2} F\left(R P_{\mathrm{in}}+I_{d}\right) \Delta f \\
\sigma_{T}^{2} & =4\left(\frac{k_{B} T}{R_{L}}\right) F_{n} \Delta f .
\end{aligned}
$$

In the above expression, $I p$ is the mean photocurrent, $M$ and $F$ are, respectively, the mean multiplication and the excess noise factor of the avalanching layer, $R$ is the QDs responsivity, $P$ in is the input optical power, $R L$ is the load resistance, $k B$ is Boltzman's constant, and $F n$ is the noise figure of the pre-amplifier. In our calculations, we assumed the following values for the above parameters: $R L=1 \mathrm{k} \Omega, T=78 \mathrm{~K}, \Delta f=10 \mathrm{~Hz}$, and $F n=2$. The dark-current data are obtained from Rotella et al. [2], according to the InAs-InGaAs DWELL detectors with and without the AlGaAs heterojunction potential-barrier layer. The dark current of a device with the current blocking layer (device 1198 in Rotella et al. [2]) at the bias of $V$ top-Vmid=-1 $\mathrm{V}$ and at the temperature of $80 \mathrm{~K}$ is $1.81 \mu \mathrm{A}$, and its value without the current-blocking layer (device 1199 in Rotella et al. [2]) under the same conditions is 23.0 $\mu \mathrm{A}$. The responsivities (when the reverse bias is $V$ top $-V$ mid $=-1 \mathrm{~V}$ ) are $0.09 \mathrm{~W} / \mathrm{A}$ and $0.14 \mathrm{~A} / \mathrm{W}$ for device 1198 and 1199, respectively.

As for the avalanche multiplication factor $M$ and the excess noise factor $F$ we used the values generated by the dead-space-multiplication-theory (DSMT) [8] model applied to a 200-nm GaAs multiplication layer. The computed excess noise factor, as a function of the mean gain, is shown in Fig. 5. 
A. Results

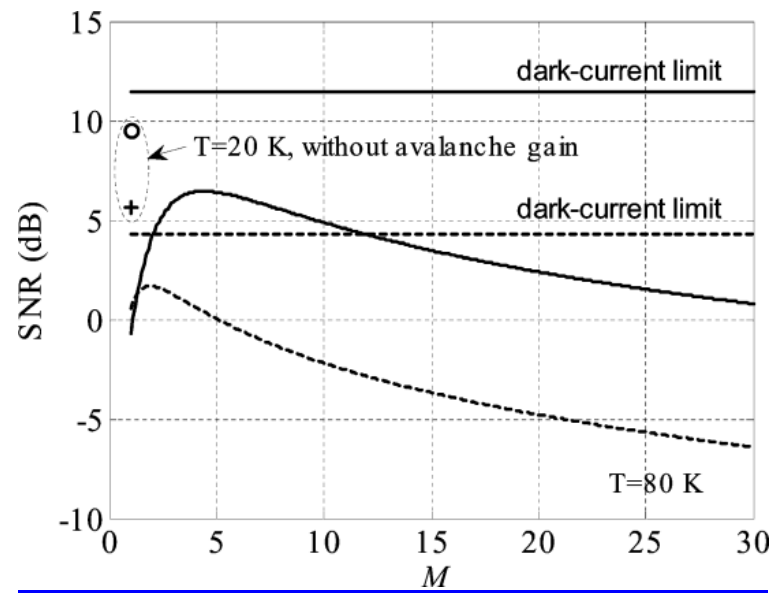

Fig. 6. Calculated signal-to-noise ratio (SNR) at $T=80 \mathrm{~K}$, as a function of the avalanche mean gain, $M$, for QDAPs with (solid) and without (dashed) the heterojunction potential-barrier layer. In both cases, the QD sections are biased at $V$ top $-V$ mid $=-1 \mathrm{~V}$; the bias on the avalanche photodiode section of the QDAP is $V$ mid- $V$ bot $=-9.2 \mathrm{~V}$ for the device with the heterojunction potential-barrier layer and $V$ mid-Vbot=-7.9 $V$ for the device without the barrier layer. The straight lines represent the dark-current-limited SNR $(M=1$ and $\sigma 2 T=0)$ at $T=80 \mathrm{~K}$ for the case with (solid) and without (dashed) the heterojunction potential-barrier layer. The circle (o) and cross (+) symbols represent the SNR at $T=20 \mathrm{~K}$ for unity-gain QDAPs with and without the heterojunction potential-barrier layer, respectively. Note that the peak SNR for the QDAP with the heterojunction potential-barrier layer at $T=80 \mathrm{~K}$ (peak of the solid curve) exceeds the SNR value at $T=20 \mathrm{~K}$ with unity avalanche gain (the " + " symbol). Thus, the optimal utilization of the avalanche gain results in a $60-\mathrm{K}$ cooling advantage in the example considered.

Fig. 6 shows the calculated SNR as a function of the APDs mean gain $M$ for an operating temperature of $80 \mathrm{~K}$. The value of the incident power Pin was set to $100 \mathrm{pW}$ (corresponding to an irradiance from a 300-K scene in the 8-12- $\mu \mathrm{m}$ band under F2 illumination on a $20 \times 20 \mu \mathrm{m}$ pixel). This would, for example, yield a 9-pA photocurrent in the QDIP section of the QDAP with the heterojunction potential-barrier layer. Consider first the device with the heterojunction potential-barrier layer. The reverse bias applied to the QD section is again Vtop $-V \mathrm{mid}=-1 \mathrm{~V}$, which is one of the operating biases used by Rotella et al. [2] As expected, Johnson noise causes severe degradation in the SNR in the absence of multiplication gain (i.e., at $M=1$ ), as seen by comparing the initial point of the solid curve, at which SNR=0.85, to its corresponding dark-current-limited level for which $S N R \approx 14$, shown by the horizontal solid line. Recall that the dark-current-limited SNR assumes no Johnson noise and no excess noise; it corresponds to the special case when $M=1$ and $\sigma 2 T=0$ in (2) and it represents the best performance possible in the absence of Johnson noise. As the avalanche gain increases, so does the SNR, peaking at an optimal value for the gain and decreasing beyond it due to the dominance of the excess noise associated with avalanche multiplication. In the case of the QDAP with the heterojunction potential-barrier layer, the peak SNR, which occurs at an avalanche gain of 4.4, is approximately $32 \%$ of the dark-current-limited SNR for the operating temperature of $80 \mathrm{~K}$. This amounts to an improvement in the SNR by a factor of five, compared to the case when no gain is introduced. The SNR-optimizing gain of 4.4 corresponds to an electric field of approximately 460 $\mathrm{kV} / \mathrm{cm}$ in the 200-nm GaAs avalanche layer, thereby requiring the application of reverse bias of Vmid-Vbot=-9.2 V. We emphasize that in the proposed three-terminal QDAP structure, the bias across the QD and APD sections can be controlled independently.

A similar behavior is seen for the device without the heterojunction potential-barrier layer, as seen from the dashed curve and line in Fig. 6 . In this case, the peak SNR, which occurs at an avalanche gain of about 2, is approximately $55 \%$ of the dark-current-limited SNR. In this case, we obtain an improvement in the SNR by a factor of 1.3, compared to the case when no gain is introduced. As the dark-current-limited SNR of the QDAP 
with the heterojunction potential-barrier layer is higher than that for a QDAP without the barrier, the gainoptimized SNR is expected to be higher in the device with the heterojunction barrier.

It is also seen from Fig. 6 that the SNR of a device without the heterojunction potential-barrier layer is higher than that for the device with the barrier when $M=1$. This behavior is contrary to that exhibited by the darkcurrent-limited SNR (i.e., in the absence of Johnson noise), and it is due to the combined effects of Johnson noise (which dominates the dark-current noise in our example) and the reduced responsivity in the device with the heterojunction potential-barrier layer. Thus, in the absence of any avalanche gain, the detrimental effect of Johnson noise is more severe on the device with the potential-barrier layer compared to the device that does not have the barrier layer. However, once the multiplication gain increases (i.e., $M>2$ ), the SNR of the device with the barrier begins to exceed that of the device without the barrier layer. Namely, in the presence of Johnson noise, the avalanche gain works to restore the dark-current-limited SNR advantage of the device with the heterojunction potential-barrier layer.

Finally, the SNR enhancement offered by the avalanche gain can be exploited to relax cooling requirements. A gain-optimized QDAP can be operated at substantially higher temperatures in comparison to a QD detector (or equivalently, compared to a QDAP with $M=1$ ). For example, for the QDAP with the heterojunction potentialbarrier layer, an SNR of 3.7 can be achieved at $T=20 \mathrm{~K}$ and $M=1$ (see Fig. 6). At $T=80 \mathrm{~K}$, on the other hand, the peak SNR, i.e., when $M=4.4$, is over 4.4. In other words, for the Johnson-noise level considered (in this example the standard deviation, $\sigma T$, of the Johnson noise is approximately four times greater than the dark current), the avalanche gain can be used to relax the operating temperature by over $60 \mathrm{~K}$.

\section{SECTION IV. Conclusion}

We proposed a novel device, called the QDAP, which combines an intersubband quantum-dot detector with an avalanche photodiode to increase the SNR of the photocurrent in the presence of Johnson noise. The QDAP is a three-terminal device for which the biases of the quantum-dot and avalanche-photodiode sections can be controlled independently. This is a desirable feature as it: 1) facilitates optimizing the avalanche gain to maximize the SNR when operated in a linear mode while maximizing the quantum-dot responsivity and 2) permits operating the device in Geiger-mode by increasing the reverse-bias of the APD section beyond avalanche breakdown. The QDAP can either achieve a higher sensitivity at the same temperature or have a comparable performance at higher operational temperatures. For example, when the standard deviation $\sigma T$ of the Johnson noiseis four times the dark current, our predictions show that the SNR enhancement offered by the multiplication gain results in relaxing the cooling requirement from 20 to $80 \mathrm{~K}$. Additionally, by using a thin $(<100 \mathrm{~nm})$ avalanche multiplication layers in the APD section of the device, the operating-voltage requirements of the QDAP can be additionally reduced [7]. We believe that the value of the voltage applied to the bottom contact could be reduced to $\sim 3-5 \mathrm{~V}$ in thinner multiplication layers, thereby making the device compatible with standard readout circuits.

\section{References}

1. A. Rogalski, "Infrared detectors: Status and trends", Progr. Quantum Electron., vol. 27, pp. 59-210, 2003.

2. P. Rotella, S. Raghavan, A. Stintz, B. Fuchs, S. Krishna, C. Morath, D. Le, S. W. Kennerly, "Normal incidence InAs/InGaAs dot-in-well detectors with current blocking AlGaAs layer", J. Cryst. Growth, vol. 251, pp. 787-793, Apr. 2003.

3. O. Kwon, M. M. Hayat, S. Wang, J. C. Campbell, A. L. Holmes, B. E. A. Saleh, M. C. Teich, " Optimal excess-noise reduction in thin heterojunction $\backslash \$\{$ hbox $\{A \mid\}\} \_\{0.6\}\{\mathrm{hbox}\{\mathrm{Ga}\}\} \_\{0.4\}\{\mathrm{hbox}\{\mathrm{As}\}\} \backslash \$-G a A s$ avalanche photodiodes ", IEEE J. Quantum Electron., vol. 39, no. 10, pp. 1287-1296, Oct. 2003.

4. S. Krishna, S. Raghavan, G. von Winckel, A. Stintz, G. Ariyawansa, S. G. Matsik, A. G. U. Perera, Appl. Phys. Lett., vol. 83, pp. 2745-2747, Oct. 2003. 
5. K. A. Anselm, H. Nie, C. Hu, C. Lenox, P. Yuan, J. C. Campbell, B. G. Streetman, "Performance of thin separate absorption charge and multiplication avalanche photodiodes", J. Quantum Electron., vol. 34, no. 3, pp. 482-490, Mar. 1998.

6. M. M. Hayat, O. Kwon, S. Wang, J. C. Campbell, B. E. A. Saleh, M. C. Teich, "Boundary effects on multiplication noise in thin heterostructure avalanche photodiodes: Theory and experiment", IEEE Trans. Electron Devices, vol. 49, no. 12, pp. 2114-2123, Dec. 2002.

7. O. Kwon, M. M. Hayat, J. C. Campbell, B. E. A. Saleh, M. C. Teich, " Optimized breakdown probabilities in $\backslash\left\{\right.$ hbox $\{$ Al\}\}_\{0.6\}\{hbox $\{$ Ga $\}\} \_\{0.4\}\{$ hbox $\{$ As $\}\} \backslash \$$-GaAs heterojunction avalanche photodiodes ", IEEE Electron Device Lett., vol. 25, no. 9, pp. 599-601, Sep. 2004.

8. M. A. Saleh, M. M. Hayat, B. E. A. Saleh, M. C. Teich, "Dead-space-based theory correctrly predicts excess noise factor for thin GaAs and AIGaAs avalanche photodiodes", IEEE Trans. Electron Devices, vol. 47, no. 3, pp. 625-633, Mar. 2000. 